Published in final edited form as:

Small. 2015 April 17; 11(15): 1801-1810. doi:10.1002/smll.201403248.

\title{
Reversibly Extracellular pH Controlled Cellular Uptake and Photothermal Therapy by PEGylated Mixed-Charge Gold Nanostars
}

\section{Dr. Shouju Wang}

Department of Medical Imaging Jinling Hospital School of Medicine Nanjing University Nanjing 210002, P.R. China

State Key Laboratory of Analytical Chemistry for Life Science School of Chemistry and Chemical Engineering Nanjing University Nanjing 210093, P.R. China

\section{Dr. Zhaogang Teng}

Department of Medical Imaging Jinling Hospital School of Medicine Nanjing University Nanjing 210002, P.R. China

State Key Laboratory of Analytical Chemistry for Life Science School of Chemistry and Chemical Engineering Nanjing University Nanjing 210093, P.R. China

\section{Dr. Peng Huang}

Laboratory of Molecular Imaging and Nanomedicine (LOMIN) National Institute of Biomedical Imaging and Bioengineering (NIBIB) National Institutes of Health (NIH) Bethesda, MD 20892, USA

\section{Prof. Dingbin Liu}

State Key Laboratory of Medicinal Chemical Biology Collaborative Innovation Center of Chemical Science and Engineering, and Research Center for Analytical Sciences College of Chemistry Nankai University Tianjin 300071, P.R. China

\section{Dr. Ying Liu}

Department of Medical Imaging Jinling Hospital School of Medicine Nanjing University Nanjing 210002, P.R. China

\section{Ying Tian}

Department of Medical Imaging Jinling Hospital School of Medicine Nanjing University Nanjing 210002, P.R. China

\section{Jing Sun}

Department of Medical Imaging Jinling Hospital School of Medicine Nanjing University Nanjing 210002, P.R. China

\section{Dr. Yanjun Li}

Department of Medical Imaging Jinling Hospital School of Medicine Nanjing University Nanjing 210002, P.R. China

\footnotetext{
*cjr.luguangming@vip.163.com.

Supporting Information Supporting Information is available from the Wiley Online Library or from the author.
} 


\section{Prof. Huangxian Ju}

State Key Laboratory of Analytical Chemistry for Life Science School of Chemistry and Chemical Engineering Nanjing University Nanjing 210093, P.R. China

\section{Prof. Xiaoyuan Chen}

Laboratory of Molecular Imaging and Nanomedicine (LOMIN) National Institute of Biomedical Imaging and Bioengineering (NIBIB) National Institutes of Health (NIH) Bethesda, MD 20892, USA

\section{Prof. Guangming Lu* \\ Department of Medical Imaging Jinling Hospital School of Medicine Nanjing University Nanjing 210002, P.R. China}

State Key Laboratory of Analytical Chemistry for Life Science School of Chemistry and Chemical Engineering Nanjing University Nanjing 210093, P.R. China

\section{Abstract}

Shielding nanoparticles from nonspecific interactions with normal cells/tissues before they reach and after they leave tumors is crucial for the selective delivery of NPs into tumor cells. By utilizing the reversible protonation of weak electrolytic groups to $\mathrm{pH}$ changes, long-chain amine/carboxylterminated polyethylene glycol (PEG) decorated gold nanostars (GNSs) are designed, exhibiting reversible, significant, and sensitive response in cell affinity and therapeutic efficacy to the extracellular $\mathrm{pH}(\mathrm{pHe})$ gradient between normal tissues and tumors. This smart nanosystem shows good dispersity and unimpaired photothermal efficacy in complex bioenvironment at $\mathrm{pH} 6.4$ and 7.4 even when their surface charge is neutral. One PEGylated mixed-charge GNSs with certain surface composition, GNS-N/C 4, exhibits high cell affinity and therapeutic efficacy at $\mathrm{pH} 6.4$, and low affinity and almost "zero" damage to cells at pH 7.4. Remarkably, this significant and sensitive response in cell affinity and therapeutic efficacy is reversible as local $\mathrm{pH}$ alternated. In vivo, GNS-N/C 4 shows higher accumulation in tumors and improved photothermal therapeutic efficacy than $\mathrm{pH}$-insensitive GNSs. This newly developed smart nanosystem, whose cell affinity reversibly transforms in response to $\mathrm{pHe}$ gradient with unimpaired biostability, provides a novel effective means of tumor-selective therapy.

\section{Introduction}

On-demand delivery of nanoparticles (NPs) into tumor cells remains an important challenge in the field of nanomedicine. ${ }^{[1-3]}$ Though receptor-ligand pairing is the most popularly used strategy, ${ }^{[4-9]}$ its effectiveness is greatly influenced by the heterogeneous nature of tumor and the dynamic expression of receptors during treatment. ${ }^{[10-12]}$ Hence, ongoing efforts are keenly focused on exploiting the characteristic acidic extracellular $\mathrm{pH}(\mathrm{pHe})$ of solid tumors (typically one unit of $\mathrm{pH}$ lower than that of normal tissues ${ }^{[13]}$ to trigger enhanced cellular internalization. ${ }^{[14-17]}$ The key challenge of this strategy is the complete shielding of NPs from nonspecific interaction during circulation (both before NPs reach and after NPs leave tumors into circulation). ${ }^{[1-3,18]}$ Toward this challenge, numerous efforts have been made to develop pHe-responsive strategies. Typical examples include polymeric micelles containing ionizable groups, which present demicellization at acidic environment due to strong charge 
repulsion between ionized groups and release their cargoes; Alternative strategy is shielding the cell-affinitive or positively charged moieties of NPs with acid-labile bonds, which would be hydrolyzed at tumoral $\mathrm{pHe}$ and re-emerge the shielded moieties to promote the cellular uptake of NPs in tumors. ${ }^{[4-9,19-23]}$ Although these designs offered excellent shielding of NPs before they reach tumors, few of them can re-shield NPs after they leaked out from tumors because of the irreversible nature of demicellization and bonds-hydrolysis. This limitation raises great concerns about the potential toxicity of therapeutic NPs to major organs in their following excretion process once they were activated in the slightly acidic tumors. ${ }^{[10-12,24]}$ Therefore, a reversibly pHe controlled targeting strategy that only enhances endocytosis of NPs at acidic tumoral environment and inhibits their cellular interaction both before and after pHe activation is vital in view of maximizing the tumor selectivity and minimizing the side effects of therapeutic NPs.

Functionalizing NPs with mixed-charge surfaces is one of the most effective ways to shield NPs from nonspecific interaction during circulation. ${ }^{[13,25-28]}$ However, the pH-reversible controllability over the cell affinity of mixed-charge nanoparticles (MCNPs) remains scarcely investigated. It has been reported that the net charge of MCNPs plays an important role in their interactions with cells in vitro, ${ }^{[14-17,29]}$ but the applications of MCNPs in pHeselective delivery are still hindered for two reasons. First, for MCNPs decorated with zwitterionic or oppositely charged strong electrolytic ligands, ${ }^{[26,30]}$ the net charge of MCNPs has little response to $\mathrm{pH}$ changes. Second, for MCNPs decorated with oppositely charged weak electrolytic ligands, the stability of MCNPs is rather poor when their net charge is neutralized at isoelectric point. ${ }^{[29,31]}$ The poor stability would result in rapid aggregation of MCNPs, which is obviously undesirable for the applications of NPs with well-designed shapes or structures in vivo.

Addressing these issues, we reported herein a new strategy to target tumor by reversibly controlling the cell affinity of PEGylated MCNPs as a function of pHe. We delicately decorated gold nanostars (GNSs) with long-chain amine-terminated (positively charged) and carboxyl-terminated (negatively charged) polyethylene glycol (PEG) (Scheme 1). The dense assembled PEG chains provided an additional strongly hydrophilic layer, which protected GNSs from aggregation even when their net charge was neutralized. Meanwhile, the weak electrolytic terminated groups granted reversible response in the net charge of GNSs to the change of $\mathrm{pH}$. We found that by optimizing the surface composition of PEGylated mixedcharge GNSs, a reversible, significant, and sensitive response in cell affinity to the $\mathrm{pHe}$ gradient between tumor and normal tissues could be achieved. Since our previous work showed GNSs could generate heat upon irradiation of near infrared laser, ${ }^{[32,33]}$ we could easily translate this response into therapeutic effect and perform reversibly pHe controlled photothermal therapy (PTT) for the first time.

\section{Results and Discussion}

\subsection{Synthesis and Characterization of PEGylated Mixed-Charge GNSs}

GNSs used in this study were prepared following a cetyltrimethylammonium bromide (CTAB) free method to avoid potential cytotoxicity. ${ }^{[34]}$ Transmission electron microscopy (TEM) images well illustrated the multi-branch shape of GNSs with size around $70 \mathrm{~nm}$ 
(Figure 1A and Figure S1, Supporting Information). The localized surface plasmon resonance (LSPR) of bare GNSs was at $762 \mathrm{~nm}$ (Figure S2A, Supporting Information). Dynamic light scattering (DLS) measurement showed the hydrodynamic diameter of bare GNSs was $77.5 \pm 3.2 \mathrm{~nm}$. To obtain PEGylated mixed-charge GNSs, these bare GNSs were conjugated to thiol PEG (M.W. $\approx 1000$ ) terminated with positively charged amine group $\left(\mathrm{NH}_{2}\right.$-PEG-SH) and negatively charged carboxyl group (COOH-PEG-SH) at various $\mathrm{NH}_{2} /$ $\mathrm{COOH}$ ratios $(3: 1,4: 1,5: 1,6: 1$, and 7:1). The products were purified and termed as GNSN/C 3, GNS-N/C 4, GNS-N/C 5, GNS-N/C 6, and GNS-N/C 7. The exact compositions of the two ligands assembled on the surface of GNSs were determined to be $\gamma_{\mathrm{surf}}=c_{\mathrm{NH} 2} /$ $c_{\mathrm{COOH}}=0.52,0.73,1.05,2.30,3.08$ indirectly by measuring the concentration of unbound amine and sulfhydryl groups. As controls, GNSs decorated with complete $\mathrm{NH}_{2}$-PEG-SH (GNS-NH ${ }_{2}$ ) or $\mathrm{COOH}-\mathrm{PEG}-\mathrm{SH}$ (GNS-COOH) were also synthesized. In all these experiments, the total concentration of PEG was kept constant. After PEGylation, the localized surface plasmon resonance of PEGylated GNSs slightly red shifted from 762 to $780 \mathrm{~nm}$ without significant broadening of peaks (Figure 1B and Figure S2A, Supporting Information). The relationship between the concentration of PEGylated GNSs and absorbance of LSPR was depicted in Figure S2B, Supporting Information. DLS measurements showed the increase of hydrodynamic diameter of GNSs from $77.5 \pm 3.2$ to $94.3 \pm 4.3 \mathrm{~nm}$ after PEGylation. No significant difference was observed between the sizes of GNSs decorated with various composition of PEG (Table S1, Supporting Information). Zeta potentials of PEGylated GNSs were determined in a series of buffer solutions from $\mathrm{pH} \approx 3$ to $\approx 11$. It is noteworthy that the isoelectric point of mixed-charge GNSs could be regulated continuously by adjusting the proportion of $\mathrm{NH}_{2}$-PEG-SH and COOH-PEG-SH (Figure 1C).

\section{2. pH-Independent Biostability and Photothermal Efficacy of PEGylated Mixed-Charge GNSs in Bioenvironment}

Biostability is critical for the applications of NPs in vivo. To test the biostability of PEGylated mixed-charge GNSs, we chose Dulbecco's Modified Eagle's Medium (DMEM, all DMEM used in this paper were supplemented with $10 \%$ fetal bovine serum unless otherwise stated) at $\mathrm{pH} 7.4$ and 6.4 to mimic the environment of circulation and tumor respectively. After being resuspended in DMEM, the color of PEGylated mixed-charge GNSs solutions turned from blue to dark purple at $\mathrm{pH} \mathrm{7.4,} \mathrm{or} \mathrm{to} \mathrm{yellowish} \mathrm{at} \mathrm{pH}$ 6.4. (The difference of color came from the effect of phenol red. When suspended in DMEM without phenol red, the color of PEGylated mixed-charge GNSs solutions remained blue, Figure 2A.) UV-vis measurement did not show any significant change in the shape of LSPR in GNS-N/C 3-7 solutions after three days of incubation in DMEM at pH 7.4 and 6.4 (Figure 2C). Notice that the zeta potentials of GNS-N/C 4-7 were close to zero at $\mathrm{pH} 7.4$ or 6.4, which means the long hydrophilic PEG chains can prevent neutralized GNSs from rapid aggregation. This feature was not observed in gold NPs protected by short weak electrolytic ligands without PEGylation, ${ }^{[29,35]}$ indicating the important role of PEGylation in the preservation of dispersity of MCNPs near isoelectric point.

To further understand the effect of the PEG-terminated groups to the dispersity of GNSs, we compared the biostability of GNS-N/C 4 in culture medium with that of GNSs- $\mathrm{NH}_{2}$ and GNS-COOH and GNSs decorated with a mixture of $\mathrm{NH}_{2}$-PEG-SH and mPEG-SH (GNS- 
N/M). As shown in Figure 2B, when dissolved in DMEM at pH 7.4, the color of GNS-NH solution turned to light purple, whereas other solutions appeared dark purple, indicating that aggregation occurred in GNS-NH $\mathrm{N}_{2}$ solution. After three days of incubation, the UV-vis spectra showed that both GNS-N/C 4 and GNS-COOH had excellent colloidal biostability in DMEM, while the absorbance peak of GNS-N/M slightly red shifted, and the peak of GNS$\mathrm{NH}_{2}$ almost disappeared (Figure 2D). In addition, the hydrodynamic diameters of GNS-N/C 4 and GNS-COOH were close to their initial sizes in water, whereas the hydrodynamic diameters of GNS-N/M and GNS-NH ${ }_{2}$ increased to $125.3 \pm 8.9$ and $343.2 \pm 14.8 \mathrm{~nm}$, respectively (Table S2, Supporting Information). The poor biostability of GNS-NH 2 and GNS-N/M was most likely attributed to the strong interaction between the cationic groups of NPs and serum proteins. ${ }^{[36]}$ The superior biostability of GNS-N/C 4 suggested that the assembled mixed-charge monolayer may shield the cross-linking between serum proteins and positively charged groups on NPs, providing a novel way to anchor the cell affinitive cationic moieties on the surface of NPs without sacrificing their dispersity in complex bioenvironment.

To evaluate the photothermal efficacy of PEGylated mixed-charge GNSs in bioenvironment at different $\mathrm{pH}$, the laser-heating curves of GNS-N/C 4 incubated in DMEM (pH 6.4 or 7.4) for three days were recorded. An increase of temperature up to $\approx 75^{\circ} \mathrm{C}$ was clearly observed in DMEM solutions with GNS-N/C 4 upon $808 \mathrm{~nm}$ laser irradiation at $2 \mathrm{~W} \mathrm{~cm}^{-2}$ for $3 \mathrm{~min}$ at $\mathrm{pH} 6.4$ and 7.4, while the DMEM solutions without GNSs under the same irradiation exhibited almost no change in temperature (Figure 2E). The photothermal efficacy of GNSN/C 4 was close to that of GNS-COOH due to their similar dispersity in culture medium. In stark contrast, the photothermal efficacy of GNS-N/M and GNS-NH 2 was significantly decreased after incubation in DMEM because of their insufficient biostability (Figure 2F). These results demonstrated that the mixed-charge PEG monolayer may help GNSs maintain their unique photothermal conversion capability in complex bioenvironment regardless changes in $\mathrm{pH}$, which is necessary for their therapeutic applications in vivo.

Other factors related to the photothermal efficacy of PEGylated mixed-charged GNSs were investigated. A concentration-dependent (from $2.5 \times 10^{-12} \mathrm{M}$ to $80 \times 10^{-12} \mathrm{M}$ ) and laser power-dependent (form 0.5 to $2 \mathrm{~W} \mathrm{~cm}^{-2}$ ) photothermal efficacy of GNS-N/C 4 was determined (Figure S3, Supporting Information). Furthermore, the GNSs showed superior photothermal stability over gold nanorods (GNRs) though five repeated cycles of laser heating (Figure S4, Supporting Information), which was consistent with previous report that GNSs were more resistant to melting under laser irradiation than other gold nanostructures. ${ }^{[37]}$

\section{3. pHe Controllable Cell Affinity and Therapeutic Efficacy of PEGylated Mixed-Charge GNSs}

We next investigated the cell affinity of PEGylated GNSs. Figure 3 summarizes the experimental results in which GNS-NH 2 , GNS-COOH, and GNS-N/C 3-7 were incubated with HeLa cells at pH 6.4 or 7.4 for $4 \mathrm{~h}$. The degree of cellular uptake was determined by optical microscopy and inductively coupled plasma (ICP) measurement. As shown in Figure 3A,B and Figure S5, Supporting Information, the degree of cellular uptake of GNS-N/C 4 
and $\mathbf{5}$ at $\mathrm{pH} 6.4$ is significantly higher than that at $\mathrm{pH}$ 7.4. Note that the degree of cellular uptake of GNS-NH $\mathrm{NH}_{2}$ and GNS-COOH was similar at both $\mathrm{pH} 6.4$ and 7.4, suggesting that the decrease in $\mathrm{pH}$ itself may not enhance cellular uptake by affecting cell membrane permeability. The clean background of optical microscopy images demonstrated the great biostability of PEGylated mixed-charge GNSs. In stark contrast, the GNS-NH 2 aggregated and attached to the substrate of cultural dish (Figure S6, Supporting Information). This result also suggested that the response in cell affinity of GNS-N/C $\mathbf{4}$ and $\mathbf{5}$ was less likely caused by size change due to aggregation.

To further understand the mechanism behind this response, the relationship between the zeta potential and the degree of cellular uptake of PEGylated mixed-charge GNSs was described in Figure 3C. We found that the degree of uptake depends on the zeta potential of PEGylated mixed-charge GNSs. When the zeta potential of PEGylated mixed-charge GNSs is negative $(<-10 \mathrm{mV})$, the degree of uptake is low, probably caused by the strong repulsion between the negatively charged carboxyl groups and the cell membranes. When the zeta potential is neutral $(-10$ to $+10 \mathrm{mV})$, the degree of cellular uptake remarkably increases. Numerous studies have shown that cells typically do not internalize the neutral PEGylated nanoparticles, ${ }^{[38]}$ which is not the case in our system. We suspected that the positively charged amine groups on the surface of GNSs may serve as "fuse" to trigger cellular internalization when the net charge of PEGylated GNSs is still neutral. This ability of boosting cellular uptake of neutral NPs is significantly important because the highly positively charged NPs are prone to aggregate in vivo and have several unwanted side effects, including hemolysis and platelet aggregation. ${ }^{[39]}$

To evaluate the relationship between the photothermal effect and the degree of uptake, the temperature change of HeLa cells suspensions (incubated with GNS-NH${ }_{2}$, GNS-COOH, or GNS-N/C 3-7) upon $808 \mathrm{~nm}$ laser irradiation at $2 \mathrm{~W} \mathrm{~cm}^{-2}$ was monitored. As shown in Figure S7, Supporting Information, for PEGylated mixed-charge GNSs, there was a good linear relationship between the magnitude of temperature change and the intracellular concentration of gold $\left(R^{2}=0.94\right)$. Note that the GNS-NH 2 showed higher cellular uptake than GNS-N/C 7 but similar temperature increase in cellular suspensions. We suspected that the high degree of aggregation limited its photothermal efficacy in complex bioenvironment.

To investigate the therapeutic efficacy of PEGylated mixed-charge GNSs at different $\mathrm{pH}$, HeLa cells were incubated with different concentration of GNS-NH ${ }_{2}$, GNS-COOH, or GNS$\mathrm{N} / \mathrm{C} \mathrm{3-7}$ for $4 \mathrm{~h}$ and irradiated by an $808 \mathrm{~nm}$ laser for $3 \mathrm{~min}$. The cell viability was measured by MTT assay. As shown in Figure 4, GNS-N/C $4\left(80 \times 10^{-12} \mathrm{M}\right)$ showed the greatest response to $\mathrm{pH}$ change among all PEGylated mixed-charge GNSs, promoting a drop from $83.7 \%$ viability to $5.4 \%$ when $\mathrm{pH}$ of culture medium decreased from 7.4 to 6.4 ( $p$-value $<0.001)$. This result was in line with the observation that GNS-N/C 4 turned from negative to neutral when $\mathrm{pH}$ decreased. The change in $\mathrm{pH}$ itself had no effect on the growth of HeLa cells with or without laser irradiation (Figure S8, Supporting Information), thus the response in therapeutic efficacy is unlikely induced by the $\mathrm{pH}$ decrease. GNS-NH $\mathrm{N}_{2}$ and GNS-COOH also showed similar therapeutic efficacy when $\mathrm{pH}$ altered (Figure S9, Supporting Information), further suggesting the necessary of assembled mixed-charge monolayers for pH-sensitivity. 
We also noticed that, for normal cells (293T cells), the degree of uptake of GNS-N/C 4 was remarkably lower than that of GNS- $\mathrm{NH}_{2}$ when the cells were incubated with GNSs at $\mathrm{pH}$ 7.4. After 3 min of laser irradiation, the viability of cells incubated with GNS-NH $\mathrm{N}_{2}$ dropped to $13.45 \%$, while the viability of GNS-N/C 4 treated cells maintained above $95 \%$. (Figure S10, Supporting Information). This observation further confirmed that the pHe selectivity of GNS-N/C 4 may greatly reduce the off-target photothermal damage to normal tissues.

\subsection{Reversibly pHe Controlled PTT In Vitro}

Encouraged by the response of GNS-N/C 4 to the change in $\mathrm{pH}$, this formula was chosen to explore the possibility of reversibly pHe controlled PTT. To the best of our knowledge, this is the first attempt at reversibly pHe controlled PTT. First, GNS-N/C 4 was preincubated in culture medium whose $\mathrm{pH}$ alternated from 7.4 to 6.4 for cycles and then added to cells. Considering the reversible protonation of amine/carboxyl groups, the therapeutic efficacy of GNS-N/C 4 should only be determined by the final $\mathrm{pH}$ at which the cells were cultured. As shown in Figure 5, the viability of cells after laser irradiation was above $80 \%$ when the final $\mathrm{pH}$ was 7.4 , and dropped to less than $10 \%$ when the final $\mathrm{pH}$ was 6.4 . The preincubation in culture medium of alternate $\mathrm{pH}$ had negligible effect on the therapeutic efficacy of GNS-N/C 4. The fluorescent live/dead cell staining also agreed well with the result of MTT assay. This reversible response in therapeutic efficacy may re-shield GNS-N/C 4 after they leak out from tumors, thus reduce their potential off-target damage to major organs in the following circulation.

\subsection{Biocompatibility of PEGylated Mixed-Charge GNSs}

Biocompatibility is another critical factor for the biomedical applications of NPs. To study this, GNS-NH ${ }_{2}$, GNS-COOH, and GNS-N/C 3-7 were incubated with HeLa cells, MCF-7 cells or 293T cells without exposure to laser. GNS-N/C 3-7 and GNS-COOH showed negligible cytotoxicity to both malignant (HeLa and MCF-7) and normal (293T) cells, while GNS-NH $\mathrm{N}_{2}$ showed slight cytotoxicity to $293 \mathrm{~T}$ cells (Figure 6). It is known that positive charged NPs may destruct cell membrane by inducing membrane depolarization, ${ }^{[40,41]}$ but the positively charged amine groups on the surface of PEGylated mixed-charge GNSs did not induce any significant toxicity to cells. We suspected that the negatively charged carboxyl groups may "mask" the depolarization effect of amine groups, thus improve the biocompatibility of PEGylated mixed-charge GNSs.

\subsection{Biodistribution of PEGylated Mixed-Charge GNSs and Reversibly pHe Controlled PTT In Vivo}

We finally examined the in vivo biodistribution of PEGylated mixed-charge GNSs on tumorbearing mice. Considering the potential clinical applications of PEGylated mixed-charge GNSs, orthotopic mouse model of breast cancer was established. The mice were intravenously injected with GNS-N/C 4, GNS-NH ${ }_{2}$, GNS-COOH or PBS. As shown in Figure 7A, in liver and lung, the accumulation of GNS-NH $\mathrm{NH}_{2}$ was remarkably higher than that of GNS-N/C 4 and GNS-NH $24 \mathrm{~h}$ postinjection. This observation may be explained by the positive charge and larger size of GNS-NH $\mathrm{N}_{2}$ aggregations, which make them easier to be engulfed by Kuffer's cells. As for tumors, GNS-N/C 4 showed the highest accumulation of 
$10.19 \%$ injected dose per gram (ID\%/g) of tumor, which is 1.3 times higher than that of GNS-COOH $(4.36 \% \mathrm{ID} / \mathrm{g}, p=0.024)$ and 4.8 times higher than that of $\mathrm{GNS}^{-\mathrm{NH}_{2}}(1.77 \%$ $\mathrm{ID} / \mathrm{g}, p=0.0043)$.

Since the tumor accumulation of GNSs is critical to their photothermal therapeutic efficacy, we irradiated tumors with $808 \mathrm{~nm}$ laser at $1 \mathrm{~W} \mathrm{~cm}^{-2}$ for $5 \mathrm{~min} 24 \mathrm{~h}$ postinjection. Thermal images were taken to measure the tumor temperature over the course of treatment. Mice treated with GNS-N/C 4 experienced an average $23{ }^{\circ} \mathrm{C}$ temperature increase during laser irradiation, reaching an average temperature of $56^{\circ} \mathrm{C}$ after $5 \mathrm{~min}$ of treatment. Conversely, mice treated with GNS-COOH showed a $15{ }^{\circ} \mathrm{C}$ temperature increase, and mice treated with GNS- $\mathrm{NH}_{2}$ or PBS had a temperature rise less than $8^{\circ} \mathrm{C}$ (Figure 7B,C). The therapeutic efficacy of PTT was assessed by the growth of tumor depicted in Figure 7D. The mice treated with GNS-N/C 4 showed a significant inhibition of tumor growth when compared to the mice treated with GNS-NH${ }_{2}$, GNS-COOH, and PBS, which was in line with the intense photothermal effect of this group. The therapeutic efficacy was also evaluated by haematoxylin and eosin (H\&E) staining on tumor tissue sections (Figure 7E). Extensive coagulative necrosis was observed in histological sections from group treated with GNS-N/C 4, indicating the effective destruction of tumor cells after laser irradiation. While groups treated with GNS-NH $\mathrm{N}_{2}$, GNS-COOH, and PBS showed large areas of neoplastic cells with nuclear atypia, revealing insufficient damage to the tumors.

\section{Conclusion}

We have demonstrated that GNSs with $\mathrm{pH}$-independent biostability and $\mathrm{pH}$-reversible cell affinity could be prepared by surface modification with amine/carboxyl-terminated PEG. The dense PEG chains offered strongly hydrophilic layers that protected mixed-charge GNSs from aggregation even when they are neutralized in complex bioenvironment. By optimizing the composition of amine/carboxyl-terminated PEG, GNS-N/C 4 exhibited high cell affinity and therapeutic efficacy at tumoral pHe, and low cell affinity and nearly "zero" damage at pHe of normal tissues. In mice model, intravenous injection of GNS-N/C 4 showed significantly increased tumor accumulation and complete ablation of orthotopic breast cancer xenograft under laser irradiation. Considering the excellent biocompatibility of GNS-N/C 4 and its reversal, significant, and sensitive response to $\mathrm{pHe}$ gradient, this smart nanosystem would be promising for tumor-targeted therapy and imaging.

\section{Experimental Section}

\section{Materials and Reagents}

$\mathrm{HAuCl}_{4} \cdot 3 \mathrm{H}_{2} \mathrm{O}, \mathrm{AgNO}_{3}$, sodium citrate, ascorbic acid, thiazolyl blue tetrazolium bromide (MTT), and dimethyl sulphoxide (DMSO) were purchased from Sigma-Aldrich. AminePEG-thiol $\left(\mathrm{NH}_{2}\right.$-PEG-SH, M.W. $\approx 1000 \mathrm{Da}$ ), carboxymethyl-PEG-thiol (COOH-PEG-SH, M.W. $\approx 1000 \mathrm{Da}$ ), and methoxy-PEG-thiol (mPEG-SH, M.W. $\approx 1000 \mathrm{Da}$ ) were purchased from Laysan Bio Inc. LIVE/DEAD viability/cytotoxicity kit was purchased from Invitrogen. Protein assay dye reagent was purchased from BIO-RAD. HeLa cells (human cervical cancer cells), MCF-7 cells (human breast cancer cells), 293T cells (human embryonic kidney cells), and 4T1 cells (mouse breast cancer cells) were purchased from American type 
culture collection. Dulbecco's modified eagle medium (DMEM) was prepared from dry powder (Invitrogen) with $3.7 \mathrm{~g} \mathrm{~L}^{-1} \mathrm{NaHCO}_{3}\left(\mathrm{pH} 7.4\right.$ ) or $0.37 \mathrm{~g} \mathrm{~L}^{-1} \mathrm{NaHCO} 3$ and $2.31 \mathrm{~g}$ $\mathrm{L}^{-1} \mathrm{NaCl}$ (pH 6.4) and supplemented with $10 \%$ fetal bovine serum (FBS). RPMI-1640 medium was purchased from Invitrogen. The deionized water $(\geq 18 \mathrm{M} \Omega \mathrm{cm}$ ) was obtained from a Millipore water purification system. All chemicals were analytical grade and used without further purification.

\section{Surface Modification of GNSs}

Bare GNSs were prepared by a modified seed-mediated method. To obtain PEGylated mixed-charge GNSs, a mixed solution of $\mathrm{NH}_{2}$-PEG-SH and COOH-PEG-SH was added to the solution of bare GNSs. The molar ratios of $\mathrm{NH}_{2}$-PEG-SH and COOH-PEG-SH used were $3,4,5,6$, and 7 . To obtain GNS-NH ${ }_{2}, \mathrm{NH}_{2}$-PEG-SH was added to the solution of bare GNSs. To obtain GNS-N/M, a mixed solution of $\mathrm{NH}_{2}$-PEG-SH and mPEG-SH at molar ratio of 4:1 was added to the solution of bare GNSs. To obtain GNS-COOH, the $\mathrm{pH}$ of solution of bare GNSs was firstly adjusted to $\approx 10$ by $\mathrm{NaOH}(1 \mathrm{~m})$, then COOH-PEG-SH was added. The PEG was used in large excess and their final concentration was kept constant at $\approx 37.6 \times$ $10^{-6} \mathrm{M}$ in all experiments. The solutions were kept under vigorous stir for $1 \mathrm{~h}$.

\section{Characterization of GNSs}

To determine the compositions of the two ligands on the surface of PEGylated mixed-charge GNSs, the solutions were centrifuged at $130000 \mathrm{rpm}$ for $10 \mathrm{~min}$ after stirring. The concentration of unbound sulfhydryl groups in the supernatant $\left(c_{\mathrm{freeSH}}\right)$ was measured by Ellman's test. The concentration of unbound amine group in the supernatant $\left(c_{\mathrm{freeNH} 2}\right)$ was measured via a chromogenic reaction with 2,4,6-trinitrobenzene-sulfonate (TNBS) by comparison with standard curve generated by a serious of known concentration of SH-PEG$\mathrm{NH}_{2}$. Then the surface concentration of sulfhydryl $\left(c_{\mathrm{SH}}\right)$ and amine groups $\left(c_{\mathrm{NH} 2}\right)$ was calculated by subtracting $c_{\text {freeSH }}$ and $c_{\text {freeNH} 2}$ from initial concentration of sulfhydryl and amine groups. The compositions of the two ligands on the surface of PEGylated mixedcharge GNSs were calculated as $\gamma_{\mathrm{surf}}=c_{\mathrm{NH} 2} / c_{\mathrm{COOH}}=c_{\mathrm{NH} 2} /\left(c_{\mathrm{SH}^{-}} c_{\mathrm{NH} 2}\right)$.

To characterize the physical properties of PEGylated GNSs, these GNSs were centrifuged, washed $\left(3 \times 10 \mathrm{~mL} \mathrm{H}_{2} \mathrm{O}\right)$, and resuspended in pure water. The morphology of GNSs was recorded by a JEOL JEM-2100 transmission electronic microscope (Japan). Hydrodynamic diameter and Zeta potential were measured with Zeta/PALS particle size and surface potential analyzer (Brookhaven Instruments). For Zeta potential measurement, the PEGylated GNSs were diluted in 0.1X PBS whose pH was titrated by $1 \mathrm{~m} \mathrm{NaOH}$ or $1 \mathrm{~m} \mathrm{HCl}$ to $3.1,5.2,6.4,7.4,8.7,9.9$, and 10.9. The gold concentration in solution was determined by inductively coupled plasma-atomic emission spectroscopy (ICP-AES) using a PerkinElmer Optima-5300DV spectrometer. The corresponding concentration of GNSs was calculated by a sphere model of $70 \mathrm{~nm}$ diameter. ${ }^{[32]} \mathrm{UV}$-vis spectra were measured by a Lambda $35 \mathrm{UV}-$ vis spectrophotometer (PerkinElmer Instruments). For phantom studies of photothermal effect of PEGylated GNSs, $1 \mathrm{~mL}$ of GNS-N/C 4 solution (concentration from $2.5 \times 10^{-12} \mathrm{M}$ to $80 \times 10^{-12} \mathrm{M}$ ) was irradiated by an $808 \mathrm{~nm}$ laser (power density from $0.5 \mathrm{~W} \mathrm{~cm}^{-2}$ to $2 \mathrm{~W}$ $\mathrm{cm}^{-2}$ ) for $3 \mathrm{~min}$. For phantom studies of photothermal stability of gold nanostructures, $1 \mathrm{~mL}$ of GNS-N/C 4 solution $\left(80 \times 10^{-12} \mathrm{M}, \approx 0.7 \mathrm{OD}\right)$ was irradiated by an 808 laser $\left(2 \mathrm{~W} \mathrm{~cm}^{-2}\right)$ 
for five ON-OFF cycles (ON: $1.5 \mathrm{~min}$, OFF: $15.5 \mathrm{~min}$ ). The excitation source was an 808 nm diode-pumped solid-state laser system (Hi-Tech Optoelectronics Co., Ltd, China). The thermal images were captured by a MAGNITY f15F1 infrared camera (Wuhan VST Light \& Technology Co., Ltd, China).

\section{Cellular Uptake Studies}

HeLa cells were cultured in DMEM supplemented with 10\% FBS in 6-well plate at $\mathrm{pH} 7.4$ or 6.4 followed by addition of PEGylated GNSs suspended in DMEM solutions. The final concentration of PEGylated GNSs (GNS-NH 2 , GNS-COOH, GNS-N/C 3-7) was $80 \times 10^{-12}$ M. After incubation at $37{ }^{\circ} \mathrm{C}$ and $5 \% \mathrm{CO}_{2}$ for $4 \mathrm{~h}$, cells were visualized by bright field microscopy. Cells were then detached from the substrate with trypsin, moved to separate vials, centrifuged and dispensed in $1.2 \mathrm{~mL}$ PBS. $0.2 \mathrm{~mL}$ of this suspension was collected and used to determine the amount of protein by Bradford method. Then the suspension was digested by freshly prepared aqua regia following by dilution with $2 \% \mathrm{HNO}_{3}$. The amount of gold was determined by ICP-AES. The uptake of GNSs was calculated as the ratio of the weight of gold to the weight of protein.

\section{In Vitro PTT Studies}

HeLa cells were cultured in DMEM supplemented with 10\% FBS in 96-well plate at pH 7.4 or 6.4 followed by addition of PEGylated GNSs suspended in DMEM solutions. The final concentration of PEGylated GNSs (GNS-NH ${ }_{2}$, GNS-COOH, GNS-N/C 3-7) was $10 \times$ $10^{-12}, 20 \times 10^{-12}, 40 \times 10^{-12}, 60 \times 10^{-12}$, and $80 \times 10^{-12} \mathrm{M}$. After incubation at $37^{\circ} \mathrm{C}$ and $5 \% \mathrm{CO}_{2}$ for $4 \mathrm{~h}$, cells were washed by cold PBS for three times and irradiated by an $808 \mathrm{~nm}$ laser at $2 \mathrm{~W} \mathrm{~cm}^{-2}$ for $3 \mathrm{~min}$. The cells were then incubated for $2 \mathrm{~h}$ with DMEM containing $250 \mu \mathrm{g} \mathrm{mL}-1$ of MTT. After discarding the culture medium, $200 \mu \mathrm{L}$ of DMSO was added to dissolve the precipitates, and the resulting solution was measured for absorbance at $570 \mathrm{~nm}$. To exclude the interference from the absorption of GNSs, the final absorbance value was adjusted to calculate cellular viability by subtracting the absorbance of background (cells underwent the same treatment but without incubation with MTT).

\section{Reversibly pHe Controlled PTT In Vitro}

HeLa cells were cultured in DMEM supplemented with 10\% FBS in 96-well plate at pH 7.4 or 6.4. GNS-N/C 4 was preincubated in DMEM whose $\mathrm{pH}$ alternated from 7.4 to 6.4 for cycles. In detail, cycle 1: GNS-N/C 4 suspended in DMEM (pH 7.4) was added to HeLa cells cultured at $\mathrm{pH}$ 7.4; cycle 2: GNS-N/C 4 suspended in DMEM (pH 6.4) was added to HeLa cells cultured at $\mathrm{pH}$ 6.4; cycle 3: GNS-N/C 4 was pre-incubated in DMEM (pH 6.4) for $1 \mathrm{~h}$, then centrifuged, resuspended in DMEM ( $\mathrm{pH} 7.4$ ), and added to HeLa cells cultured at $\mathrm{pH}$ 7.4; cycle 4: GNS-N/C 4 was preincubated in DMEM (pH 7.4) for $1 \mathrm{~h}$, then centrifuged, resuspended in DMEM (pH 6.4) and added to HeLa cells cultured at $\mathrm{pH}$ 6.4; cycle 5: GNS-N/C 4 was pre-incubated in DMEM ( $\mathrm{pH} 7.4$ ) for $1 \mathrm{~h}$, then centrifuged and incubated in DMEM (pH 6.4) for $1 \mathrm{~h}$. After that, these GNSs were centrifuged, resuspended in DMEM (pH 7.4), and added to HeLa cells cultured at $\mathrm{pH} 7.4$; cycle 6: GNS-N/C 4 was preincubated in DMEM ( $\mathrm{pH}$ 6.4) for $1 \mathrm{~h}$, then centrifuged and incubated in DMEM (pH 7.4) for $1 \mathrm{~h}$. After that, these GNSs were centrifuged and incubated in DMEM (pH 6.4) and 
added to HeLa cells cultured at $\mathrm{pH}$ 6.4. In all cycles, the final concentration of GNS-N/C 4 was $\approx 80 \times 10^{-12} \mathrm{M}$. After incubation with cells at $37^{\circ} \mathrm{C}$ and $5 \% \mathrm{CO}_{2}$ for $4 \mathrm{~h}$, cells were washed by cold PBS for three times and irradiated by an $808 \mathrm{~nm}$ laser at $2 \mathrm{~W} \mathrm{~cm}^{-2}$ for 3 min. The cell viability was determined by MTT assay as protocol described in the previous section. Cell live/dead staining was performed following the manufacturer's instruction (Invitrogen). The fluorescence imaging was captured by an IX71 microscopy (Olympus).

\section{Biocompatibility Studies}

HeLa cells, MCF-7 cells and 293T cells were cultured in DMEM supplemented with 10\% FBS in 96-well plate at pH 7.4 followed by addition of PEGylated GNSs suspended in DMEM solutions. The final concentration of PEGylated GNSs $\left(\mathrm{GNS}^{-\mathrm{NH}_{2}}\right.$, GNS-COOH, GNS-N/C 3-7) was $80 \times 10^{-12} \mathrm{M}$. After incubation at $37^{\circ} \mathrm{C}$ and $5 \% \mathrm{CO}_{2}$ for $4 \mathrm{~h}$ or $24 \mathrm{~h}$, the cell viability was determined by MTT assay as protocol described in the previous section.

\section{In Vivo Biodistribution and PTT Studies}

All procedures involving animals were approved by the Institutional Animal Care and Use Committee of Jinling hospital. Female Balb/c mice (6-8 weeks old) were maintained in a pathogen-free environment. 4T1 cells were cultured in RPMI-1640 medium supplemented with 10\% FBS. Orthotopic mice models of breast cancer were established by injection of $4 \mathrm{~T} 1$ cells $\left(1 \times 10^{5}\right)$ into the fat pads in right abdominal mammary gland of female Balb/c mice. When the tumor size reached $\approx 60 \mathrm{~mm}^{3}$, the mice were subjected to four different treatments: group 1, intravenous (i.v.) injection of PBS following laser irradiation; group 2, i.v. injection of GNS-NH $\mathrm{N}_{2}$ following laser irradiation; group 3, i.v. injection of GNS-COOH following laser irradiation; group 4, i.v. injection of GNS-N/C 4 following laser irradiation. $100 \mu \mathrm{L}$ of $\approx 200 \times 10^{-9} \mathrm{M}$ GNS-N/C 4, GNS-COOH, GNS-NH ${ }_{2}$ or PBS were intravenously injected into the tumor-bearing mice. Tumors and major organs from mice of group 2, 3, and 4 were collected $24 \mathrm{~h}$ post-injection (three mice per group) and washed by PBS. The tissues were then digested by freshly prepared aqua regia to determine the gold concentration by ICP-AES.

For PTT studies, the tumors were exposed to $808 \mathrm{~nm}$ irradiation at $1 \mathrm{~W} \mathrm{~cm}^{-2}$ for $5 \mathrm{~min} 24 \mathrm{~h}$ post-injection. Tumors from the four groups (one mouse per group) were harvested six days after treatment for histological study by haematoxylin-eosin (H\&E) staining. The volumes of tumors were monitored by caliper every two days after treatment and calculated by its greatest longitudinal diameter (length) and greatest transverse diameter (width) as this equation: volume $=$ length $\times$ width $^{2} \times 0.5$ (six mice per group).

In total, 40 mice were used for animal studies, including 12 mice for biodistribution studies, 24 mice for tumor growth studies, and 4 mice for H\&E staining studies.

\section{Statistical Analysis}

The difference in cellular uptake and photothermal therapeutic efficacy of PEGylated GNSs between pH 6.4 and 7.4 was analyzed with two-sample t-test. The difference in biodistribution and tumor growth inhibition effect among GNS-COOH, GNS-NH 2 , and 
GNS-N/C 4 was analyzed with ANOVA followed by post hoc TukeyHSD test. All statistical analysis was performed using R statistical software (3.0.1).

\section{Supplementary Material}

Refer to Web version on PubMed Central for supplementary material.

\section{Acknowledgements}

S.W. and T.Z. contributed equally to this work. This project is financially supported by the National Key Basic Research Program of the P.R. China (Program Nos. 2014CB744504 and 2011CB707700), the Major International (Regional) Joint Research Program of China (Program No. 81120108013), the National Natural Science Foundation of China (Program Nos. 30930028 and 81201175), and the Natural Science Foundation of Jiangsu Province (Program No. BK20140734).

\section{References}

[1]. Thakor AS, Gambhir SS. Ca-Cancer J. Clin. 2013; 63:395. [PubMed: 24114523]

[2]. Smith BR, Ghosn EEB, Rallapalli H, Prescher JA, Larson T, Herzenberg LA, Gambhir SS. Nat. Nanotechnol. 2014; 9:481. [PubMed: 24727688]

[3]. Lee D-E, Koo H, Sun I-C, Ryu JH, Kim K, Kwon IC. Chem. Soc. Rev. 2012; 41:2656. [PubMed: 22189429]

[4]. Huang P, Bao L, Zhang C, Lin J, Luo T, Yang D, He M, Li Z, Gao G, Gao B, Fu S, Cui D. Biomaterials. 2011; 32:9796. [PubMed: 21917309]

[5]. Wang J, Zhu G, You M, Song E, Shukoor MI, Zhang K, Altman MB, Chen Y, Zhu Z, Huang CZ, Tan W. ACS Nano. 2012; 6:5070. [PubMed: 22631052]

[6]. Gao J, Chen K, Xie R, Xie J, Yan Y, Cheng Z, Peng X, Chen X. Bioconjugate Chem. 2010; 21:604.

[7]. Lee S, Xie J, Chen X. Chem. Rev. 2010; 110:3087. [PubMed: 20225899]

[8]. Smith BR, Zavaleta C, Rosenberg J, Tong R, Ramunas J, Liu Z, Dai H, Gambhir SS. Nano Today. 2013; 8:126.

[9]. Zhou B, Li J, Feng B-J, Ouyang Y, Liu Y-N, Zhou F. J. Inorg. Biochem. 2012; 116:19. [PubMed: 23010325]

[10]. Bedard PL, Hansen AR, Ratain MJ, Siu LL. Nature. 2013; 501:355. [PubMed: 24048068]

[11]. Huang X, Peng X, Wang Y, Wang Y, Shin DM, El-Sayed MA, Nie S. ACS Nano. 2010; 4:5887. [PubMed: 20863096]

[12]. Marusyk A, Almendro V, Polyak K. Nat. Rev. Cancer. 2012; 12:323. [PubMed: 22513401]

[13]. Gerweck LE, Seetharaman K. Cancer Res. 1996; 56:1194. [PubMed: 8640796]

[14]. Du J-Z, Mao C-Q, Yuan Y-Y, Yang X-Z, Wang J. Biotechnol. Adv. 2014; 32:789. [PubMed: 23933109]

[15]. Mura S, Nicolas J, Couvreur P. Nat Mater. 2013; 12:991. [PubMed: 24150417]

[16]. Liu J, Huang Y, Kumar A, Tan A, Jin S, Mozhi A, Liang X-J. Biotechnol. Adv. 2014; 32:693. [PubMed: 24309541]

[17]. Min KH, Kim J-H, Bae SM, Shin H, Kim MS, Park S, Lee H, Park R-W, Kim I-S, Kim K, Kwon IC, Jeong SY, Lee DS. J. Controlled Release. 2010; 144:259.

[18]. Gullotti E, Yeo Y. Mol. Pharm. 2009; 6:1041. [PubMed: 19366234]

[19]. Yuan Y-Y, Mao C-Q, Du X-J, Du J-Z, Wang F, Wang J. Adv. Mater. 2012; 24:5476. [PubMed: 22886872]

[20]. Huang S, Shao K, Liu Y, Kuang Y, Li J, An S, Guo Y, Ma H, Jiang C. ACS Nano. 2013; 7:2860. [PubMed: 23451830]

[21]. Jin E, Zhang B, Sun X, Zhou Z, Ma X, Sun Q, Tang J, Shen Y, Van Kirk E, Murdoch WJ, Radosz M. J. Am. Chem. Soc. 2013; 135:933. [PubMed: 23253016] 
[22]. Du J-Z, Du X-J, Mao C-Q, Wang J. J. Am. Chem. Soc. 2011; 133:17560. [PubMed: 21985458]

[23]. Ling D, Park W, Park S-J, Lu Y, Kim KS, Hackett MJ, Kim BH, Yim H, Jeon YS, Na K, Hyeon T. J. Am. Chem. Soc. 2014; 136:5647. [PubMed: 24689550]

[24]. Velema WA, Szymanski W, Feringa BL. J. Am. Chem. Soc. 2014; 136:2178. [PubMed: 24456115]

[25]. Yang W, Zhang L, Wang S, White AD, Jiang S. Biomaterials. 2009; 30:5617. [PubMed: 19595457]

[26]. Jiang S, Cao Z. Adv. Mater. 2010; 22:920. [PubMed: 20217815]

[27]. Yang W, Xue H, Li W, Zhang J, Jiang S. Langmuir. 2009; 25:11911. [PubMed: 19583183]

[28]. Murthy AK, Stover RJ, Hardin WG, Schramm R, Nie GD, Gourisankar S, Truskett TM, Sokolov KV, Johnston KP. J. Am. Chem. Soc. 2013; 135:7799. [PubMed: 23565806]

[29]. Pillai PP, Huda S, Kowalczyk B, Grzybowski BA. J. Am. Chem. Soc. 2013; 135:6392. [PubMed: 23527630]

[30]. Liu X, Huang H, Jin Q, Ji J. Langmuir. 2011; 27:5242. [PubMed: 21476529]

[31]. Liu X, Chen Y, Li H, Huang N, Jin Q, Ren K, Ji J. ACS Nano. 2013; 7:6244. [PubMed: 23799860]

[32]. Wang S, Huang P, Nie L, Xing R, Liu D, Wang Z, Lin J, Chen S, Niu G, Lu G, Chen X. Adv. Mater. 2013; 25:3055. [PubMed: 23404693]

[33]. Nie L, Wang S, Wang X, Rong P, Ma Y, Liu G, Huang P, Lu G, Chen X. Small. 2014; 10:1585. [PubMed: 24150920]

[34]. Yuan H, Khoury CG, Hwang H, Wilson CM, Grant GA, Vo-Dinh T. Nanotechnology. 2012; 23:075102. [PubMed: 22260928]

[35]. Nam J, Won N, Jin H, Chung H, Kim S. J. Am. Chem. Soc. 2009; 131:13639. [PubMed: 19772360]

[36]. Nel AE, Mädler L, Velegol D, Xia T, Hoek EMV, Somasundaran P, Klaessig F, Castranova V, Thompson M. Nat. Mater. 2009; 8:543. [PubMed: 19525947]

[37]. Wang Y, Black KC, Luehmann H, Li W, Zhang Y, Cai X, Wan D, Liu S-Y, Li M, Kim P. ACS Nano. 2013; 7:2068. [PubMed: 23383982]

[38]. Verma A, Stellacci F. Small. 2010; 6:12. [PubMed: 19844908]

[39]. Albanese A, Tang PS, Chan WCW. Annu. Rev. Biomed. Eng. 2012; 14:1. [PubMed: 22524388]

[40]. Arvizo RR, Miranda OR, Thompson MA, Pabelick CM, Bhattacharya R, Robertson JD, Rotello VM, Prakash YS, Mukherjee P. Nano Lett. 2010; 10:2543. [PubMed: 20533851]

[41]. Goodman CM, McCusker CD, Yilmaz T, Rotello VM. Bioconjugate Chem. 2004; 15:897. 

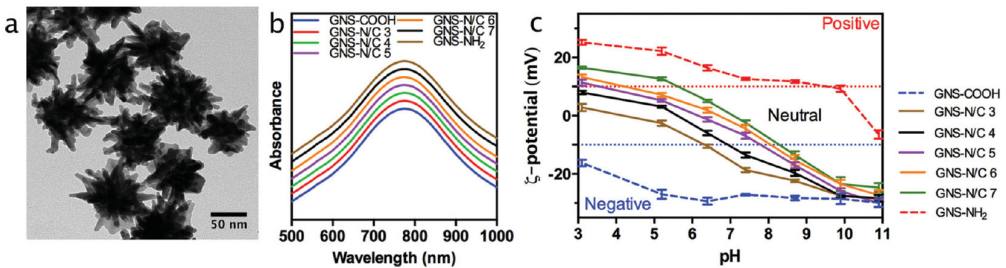

Figure 1.

(A) Typical transmission electron microscopic image of GNSs. Scale bar: $50 \mathrm{~nm}$. B) UV-vis spectra of GNS-NH ${ }_{2}$, GNS-COOH, and GNS-N/C 3-7. C) Zeta potentials of GNS-NH $\mathrm{N}_{2}$, GNS-COOH, and GNS-N/C 3-7 in a series of buffer solutions from $\mathrm{pH} \approx 3$ to $\approx 11$. 


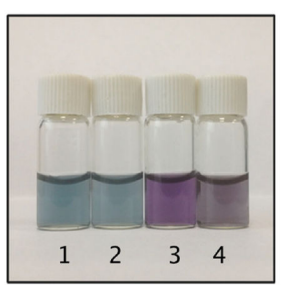

C

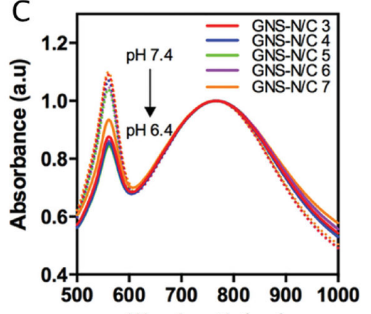

Wavelength (nm)

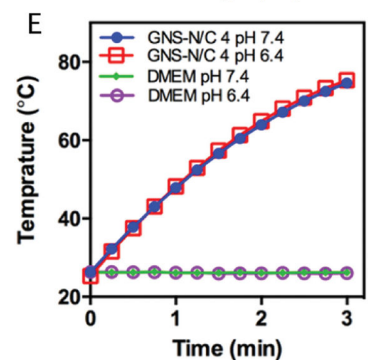

B
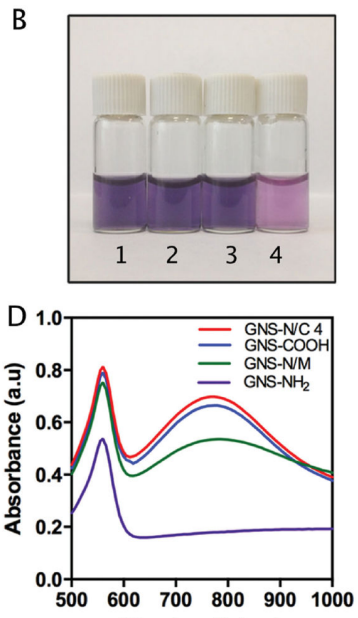

Wavelength (nm)

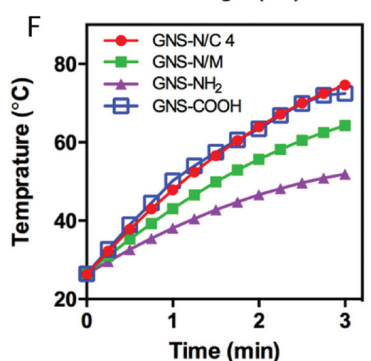

Figure 2.

A) Digital photos of GNS-N/C 4 suspended in $\mathrm{H}_{2} \mathrm{O}$ (1), DMEM at $\mathrm{pH} 7.4$ without phenol red (2), DMEM at pH 7.4 (3), and DMEM at pH 6.4 (4). B) Digital photos of GNS-N/C 4 (1), GNS-COOH (2), GNS-N/M (3), and GNS-NH 2 (4) suspended in DMEM at pH 7.4. C) Normalized UV-vis spectra of GNS-N/C 3-7 incubated in DMEM at pH 7.4 (dashed lines) or 6.4 (solid lines) after 3 days. The peaks at $560 \mathrm{~nm}$ come from phenol red. D) UV-vis spectra of GNS-N/C 4, GNS-COOH, GNS-N/M, and GNS-NH $\mathrm{N}_{2}$ incubated in DMEM at $\mathrm{pH}$ 7.4 after three days. E) Heating curves of DMEM at pH 7.4 or 6.4 with or without GNS-N/C 4 upon $808 \mathrm{~nm}$ laser irradiation ( $2 \mathrm{~W} \mathrm{~cm}^{-2}, 3 \mathrm{~min}$ ). GNS-N/C 4 was incubated in DMEM for three days. F) Heating curves of GNS-N/C 4, GNS-N/M, GNS-COOH, and GNS-NH upon $808 \mathrm{~nm}$ laser irradiation $\left(2 \mathrm{~W} \mathrm{~cm}{ }^{-2}, 3 \mathrm{~min}\right.$ ) after incubation in DMEM for three days. 

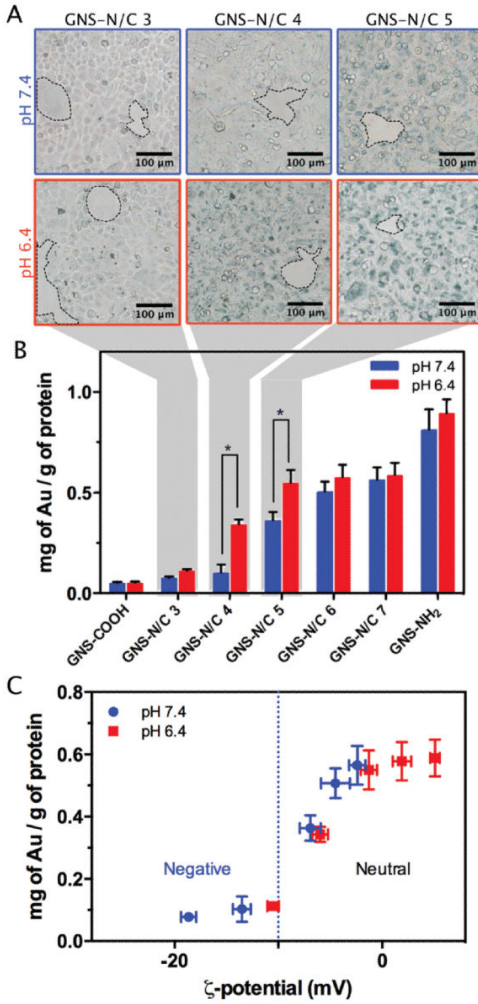

Figure 3.

A) Optical microscopy images of HeLa cells after incubation with $80 \times 10^{-12} \mathrm{M}$ GNS-N/C 3-5 for $4 \mathrm{~h}$. Scale bar: $100 \mu \mathrm{m}$. The clean background circled in dashed lines indicates the excellent biostability of PEGylated mixed-charge GNSs. B) Cellular uptake of PEGylated GNSs determined by ICP after incubation with $80 \times 10^{-12} \mathrm{M} \mathrm{GNS-NH}_{2}$, GNS-COOH, and GNS-N/C 3-7 for $4 \mathrm{~h}$. Statistical difference has been indicated by asterisks. C) Plot of ICP data quantifying the degree of cellular uptake as a function of the zeta potential of PEGylated mixed-charge GNSs. 

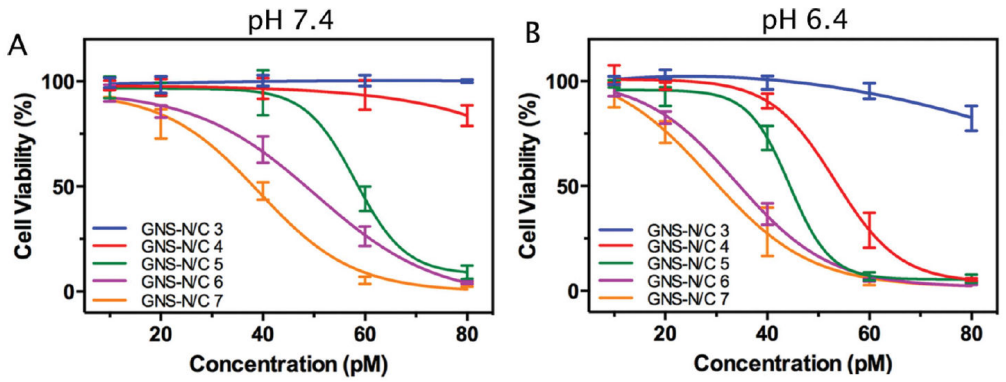

Figure 4.

Cell viability of HeLa cells incubated with different concentration of GNS-N/C 3-7 for $4 \mathrm{~h}$ at $\mathrm{pH}$ 7.4. A) or $\mathrm{pH}$ 6.4. B) after laser irradiation $\left(808 \mathrm{~nm}, 2 \mathrm{~W} \mathrm{~cm}^{-2}, 3 \mathrm{~min}\right)$. 

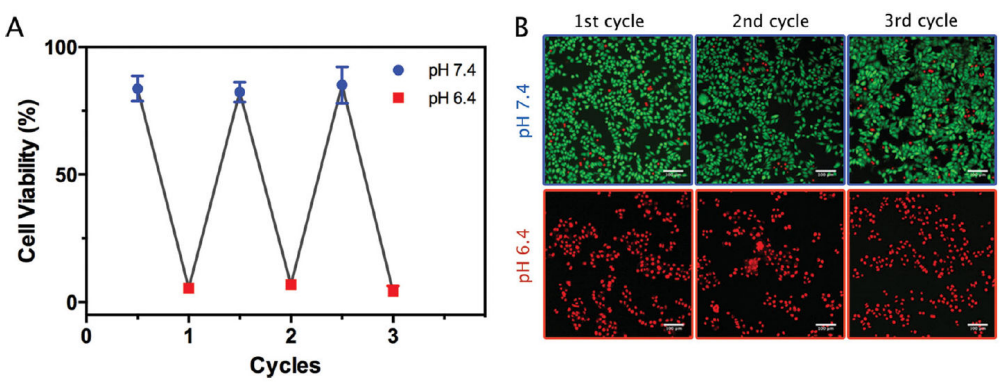

Figure 5.

A) Cell viability of HeLa cells cultured with $80 \times 10^{-12} \mathrm{M}$ GNS-N/C 4 (preincubated in DMEM whose $\mathrm{pH}$ alternated from 6.4 to 7.4 for cycles) for $4 \mathrm{~h}$ after laser irradiation (808 $\mathrm{nm}, 2 \mathrm{~W} \mathrm{~cm}{ }^{-2}, 3 \mathrm{~min}$ ). B) The corresponding fluorescence images of calcein AM (green, live cells)/propidium iodide (red, dead cells) costained HeLa cells for each cycle of $\mathrm{pH}$ reversible PTT. Scale bar: $100 \mu \mathrm{m}$. 
A

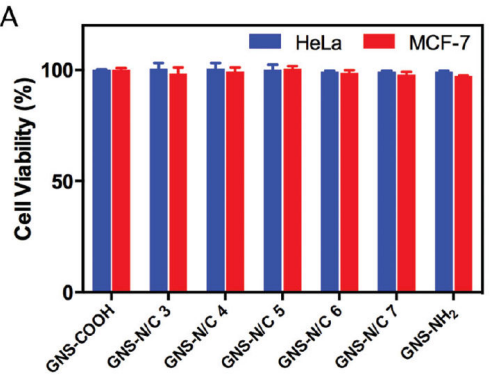

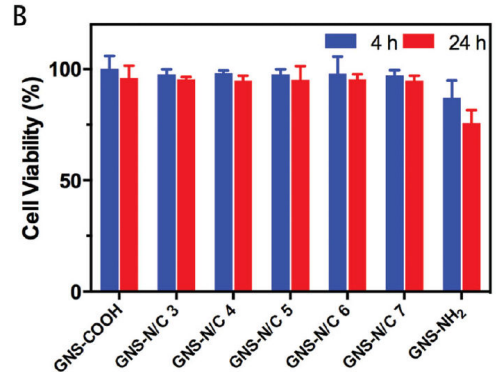

Figure 6.

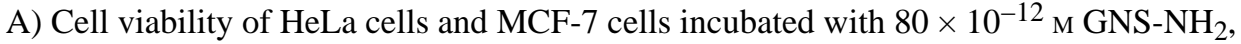
GNS-COOH, or GNS-N/C 3-7 at pH 7.4 for 24 h without laser irradiation. B) Cell viability

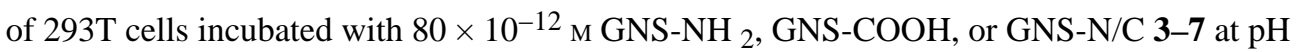
7.4 for 4 or $24 \mathrm{~h}$ without laser irradiation. 

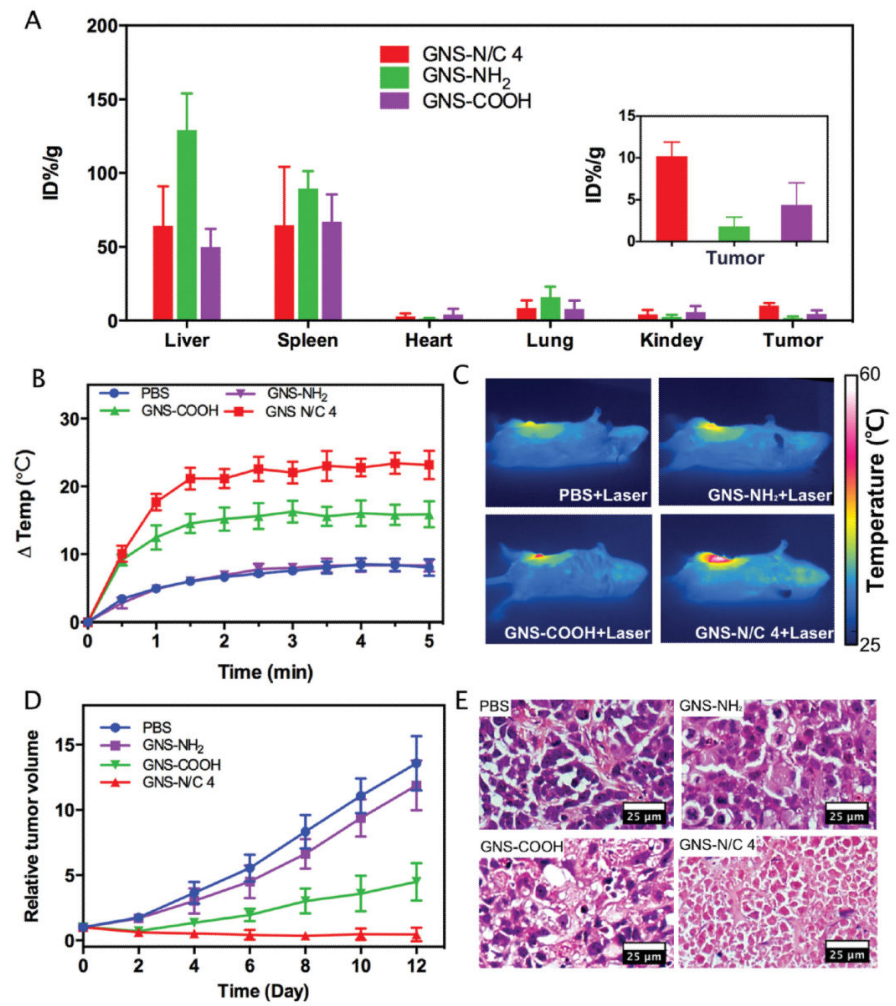

Figure 7.

A) The biodistribution of GNS-N/C 4, GNS-COOH, and GNS-NH 2 in orthotopic mouse model of breast cancer (three mice per group). B) The change in temperature of tumors upon $808 \mathrm{~nm}$ laser irradiation ( $\left.1 \mathrm{~W} \mathrm{~cm}^{-2}, 5 \mathrm{~min}\right) 24 \mathrm{~h}$ post intravenous injection of PBS, GNS$\mathrm{NH}_{2}$, GNS-COOH, or GNS-N/C 4. C) Representative thermal images of mice at the end of laser irradiation. The average starting body temperature was $33.0^{\circ} \mathrm{C}$. D) Change of relative tumor volume after $808 \mathrm{~nm}$ laser irradiation at $1 \mathrm{~W} \mathrm{~cm}^{-2}$ for $5 \mathrm{~min}$. Data are means \pm s.d. (6 mice per group). E) H\&E staining of tumor histological sections of different groups at six days after laser irradiation. Scale bar: $25 \mu \mathrm{m}$. 


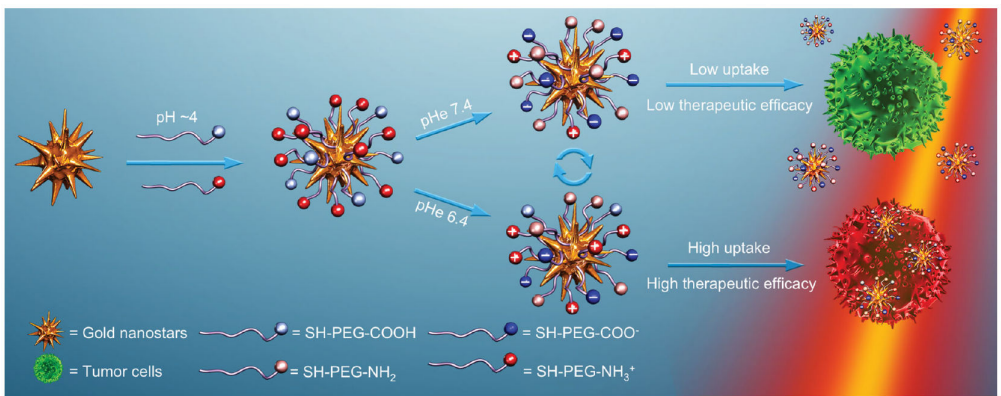

Scheme 1.

Schematic illustration of the preparation of PEGylated mixed-charge GNSs and their $\mathrm{pH}-$ reversible cell affinity and photothermal therapeutic efficacy. 